\title{
Investigation of Surface Mechanical Properties of the Copper-Solder Interface by Atomic Force Microscopy
}

\author{
Judit Kámán, Attila Bonyár, Tamás Hurtony, Gábor Harsányi \\ Department of Electronics Technology, Budapest University of Technology and Economics, Budapest, Hungary \\ kaman@ett.bme.hu
}

\begin{abstract}
In this work, the possibility to characterize the mechanical surface properties of solderjoints with AFM techniques was studied. A SAC305 solder joint cross-section was investigated with contact-mode point-spectroscopy to determine the elastic modulus, while tapping-mode imaging was used to calculate the tip-sample dissipation map. A clear advantage of these proposed methods would be the possibility to distinguish the various micro- and nanoscale components of the solder joint structure (intermetallic compounds) and measure their properties individually, which is not possible with other currently widespread methods (e.g. indentation). Our presented preliminary results show, that the mechanical properties of the $\mathrm{Cu}_{6} \mathrm{Sn}_{5}$ compound at the solder interface are closer to $\mathrm{Cu}$ compared to the much less harder Sn. The difficulties to obtain precise quantitative results with these proposed methods will also be discussed.
\end{abstract}

\section{INTRODUCTION}

In the case of the lead-free soldering technology the reliability of the solder-joints is heavily influenced by the characteristics of the intermetallic layer (IML). Its mechanical properties can be significantly different from the properties of the substrate or the tin-phase [1]. The determination of the local physical properties of the intermetallic layer may prove to be crucial for the development of better lead-free soldering technologies.

The Sn-Ag-Cu (SAC) alloys are the most commonly used lead-free substitute solders. The formed solder-joint consists of three parts; the copper substrate, the intermetallic layer and the Sn-rich phase containing the intermetallic components (IMCs). $\mathrm{Cu}_{6} \mathrm{Sn}_{5}$ is the main constituent part of the IML. This scallop type compound can spall and fragments can migrate into the Sn-rich phase [2,3]. The thickness of the IML layer and the microstructure and size of the $\mathrm{Ag}_{3} \mathrm{Sn}$ components in the Sn-rich phase can vary in the few micrometers to few hundred nanometers range.

The standard technique to investigate the elastic properties of the solder-joint is the instrumented indentation test (IIT) which causes elastic and plastic deformations in the sample with a diamond tip and these deformations can be analysed by the Oliver and Pharr method [4]. However the minimum projected area of the tip during this method is about a half micrometer, which limits the surface area that can be studied. Furthermore, during the contact with a soft sample, such as the $\beta$-Sn the pile-up effect cannot be neglected which leads to the underestimation of the contact area [5].

Thus, according to the size of the IML and IMCs, the conventional mechanical test could be problematic if we are to address these components individually. Because of this, thermal aging can be used to induce the growth of the IML layer or the measurements can be done on specifically prepared bulk samples [6]. However the real appearance of the solder components may differ from these artificially prepared forms.

AFM is a promising tool to examine the components of the cross-sectioned solder-joint in their natural size and form. It enables mechanical measurements on a nanoscale area using lower pushing forces $(\sim 100 \mathrm{nN})$ than a common nanoindentation method, so the plastic deformation of the sample can also be avoided.

There are two common methods to examine the surface mechanical properties with a basic AFM 
instrument. One is the contact-mode pointspectroscopy measurement, which can provide information about the Young's modulus at a single point. The other one is the contact-mode tapping imaging, which can allow the calculation of the energy dissipation between the tip and the surface.

\section{THEORY}

\subsection{Young's modulus determination}

During the contact-mode point-spectroscopy the tip moves towards the sample, depresses the surface and will be retracted. The so-called force-curve can be obtained from this process, which is the function of the force acting on the cantilever $(F)$ versus the tipsample distance $(\delta)$. To determine the Young's modulus $(E)$ the Hertzian model was used to fit to the elastic region of the force-curve [7]:

$$
F=\frac{2 E \tan (\alpha)}{\pi\left(1-v^{2}\right)} \delta^{2}
$$

where $\alpha$ is the half opening angle of the tip and $v$ is the Poisson-ratio.

By this model the following fitting parameter $(A$ factor) can be defined:

$$
A=\sqrt{\frac{k\left(1-v^{2}\right) \pi}{2 E \tan (\alpha)}}
$$

As it can be seen the A-factor depends on the $E$ elastic modulus through the Poisson-ratio and only the constants describing the tip. However the exact determination of these constants can be difficult.

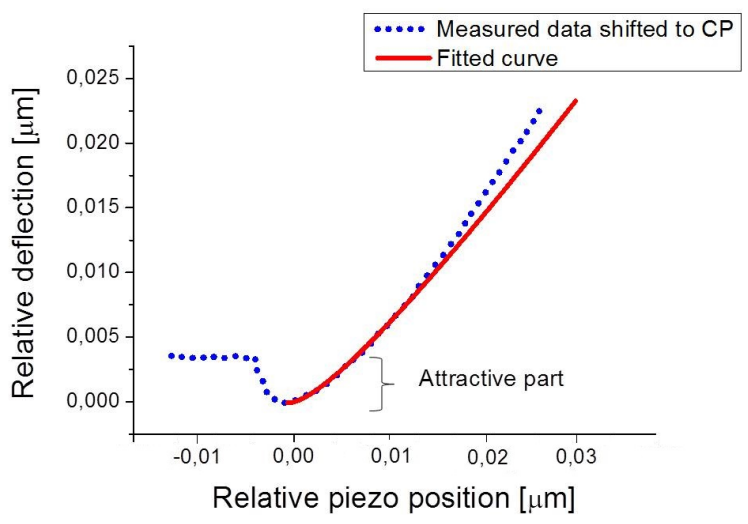

Fig. 1. Measured contact-mode point-spectroscopy data (dotted line) shifted to the contact point and fitted curve (red line) based on Eq. (1). The $(F)$ force and the $(\delta)$ tipsample distance in Eq. (1) can be derived from the relative deflection and the relative piezo position signal through a transformation.
The deviation of the A-factor is strongly depends on the correct assignment of the elastic region. However several papers were published which presented an algorithm to find the tip-sample contact point $(\mathrm{CP})$ - which could be regarded as the initial point of the fitting-less attention was paid to determine the last point of the fitting and there is no widely accepted solution for this problem $[8,9]$. In our work, considering the attractive part of the measured curves, the $\mathrm{CP}$ point was chosen as the minimum point of the curve and the last point of the fitting was determined by visual assessment (see Fig. 1).

It is important to note that the selection of the correct spring constant in accordance with the elasticity of the sample is essential to obtain relevant curves.

\subsection{Calculation of the tip-sample dissipation map}

By applying Cleveland's model and using tappingmode imaging it is possible to characterize the surface material properties on the whole scanned area.

The base of this theory is that the interaction between the tapping probe and the surface causes phase lag -besides the air damping- in the vibration of the tip compared to the drive frequency. For fixed tapping settings it was proven that the phase-lag depends on only the material properties of the components.

Cleveland et al. analyzed the energy flow in and out of the vibrating system and obtained the following equation to the average dissipation caused by tipsample interaction $[10,11]$ :

$$
\left\langle P_{t s}\right\rangle=\frac{1}{2} \frac{k A^{2} \omega_{0}}{Q}\left[\frac{A_{0}}{A} \sin \varphi-1\right]
$$

In Eq. (3) $\omega_{0}$ is the resonant frequency of the tip and $Q$ is the quality factor; both constant can be obtained from the resonance curve of the free cantilever during the tuning. $A_{0}$ is the free oscillation amplitude and $k$ is the spring constant. During the tapping-mode scanning, the feedback system tries to keep constant the $A$ amplitude and the $\varphi$ phase-lag image can be measured. So in this case the Eq. (3) shows that the power dissipation depends on only the sinus of the phase-lag, thus the calculated dissipation changes according to the material variations.

To determine the $A_{0}$ and to set the phase lag according to the model -namely match the $90^{\circ}$ in the model with the free oscillation of the cantilever - a 
tapping-mode point-spectroscopy measurement is needed, starting far enough from the sample, where the force field of the surface is negligible.

\section{Materials AND Methods}

SENJU SAC305 paste was soldered to a copper wire by a solder iron. To study the cross section of the solder joint the sample was embedded into an acryl based resin (Technovit 4006). First, wet grinding process was applied on it with the following grit sizes: 80, 320, 500 and 1200; after it was polished by 9, 3 and $1 \mu \mathrm{m}$. Finally OP-S etching was used to reveal the relevant microstructures.

Veeco (lately Bruker) diInnova type atomic force microscope was used for the measurements in contactand tapping-mode with $512 \times 512$ sampling rate and $1 \mathrm{~Hz}$ scan rate. At each measurement the PID values were optimized according to the user manual. Budget Sensors Tap150Al-G probes were used for both the tapping- and the contact-mode imaging and pointspectroscopy measurements in regard to the relatively hard components of the sample. By the datasheet of the supplier the nominal spring constant is $5 \mathrm{~N} / \mathrm{m}$, but it can have a large variation, namely between $1.5 \mathrm{~N} / \mathrm{m}$ and $15 \mathrm{~N} / \mathrm{m}$; the nominal resonant frequency is $150 \mathrm{kHz}$, however the precise values were obtained from the resonance curve during the tuning; the half opening angle of the tip $(\alpha)$ was considered as $20^{\circ}$, for the calculations $v=0.36$ Poisson-ratio was used. Gwiddion 2.27 and Mathematica 9 software were used for the data evaluation.

\section{Results AND Discussion}

\subsection{Young's modulus}

Contact-mode point-spectroscopy measurements were done on the SAC305 solder-joint cross-section to study the elastic properties of the components. Fig. 2 shows that due to the OP-S polishing during the sample preparation, the $\mathrm{Cu}$ substrate, the intermetallic layer (IML) and the spalled intermetallic components (IMC) in the solder can be clearly distinguished on the topography map.

The sharp height contrast makes the allocation of the measurement points easier and more accurate on the three investigated phases: on the $\mathrm{Cu}$ substrate, on the $\mathrm{Cu}_{6} \mathrm{Sn}_{5}$ IML layer and on the tin avoiding the
IMCs. Figure 3 presents the fitted A-factors for 30-2830 force curves at copper, IML and tin region.
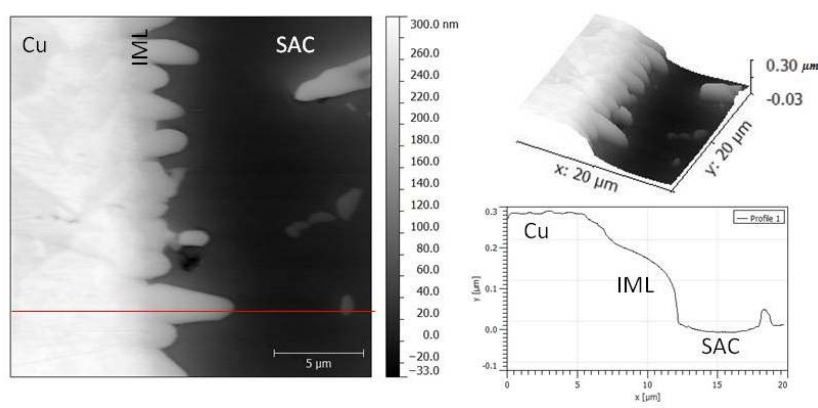

Fig. 2. $20 \mu \mathrm{m} \times 20 \mu \mathrm{m}$ contact-mode topography image of the solder-joint interface after OP-S etching (A) with 3D representation (B) and corresponding cross-section (C) at the indicated marker.
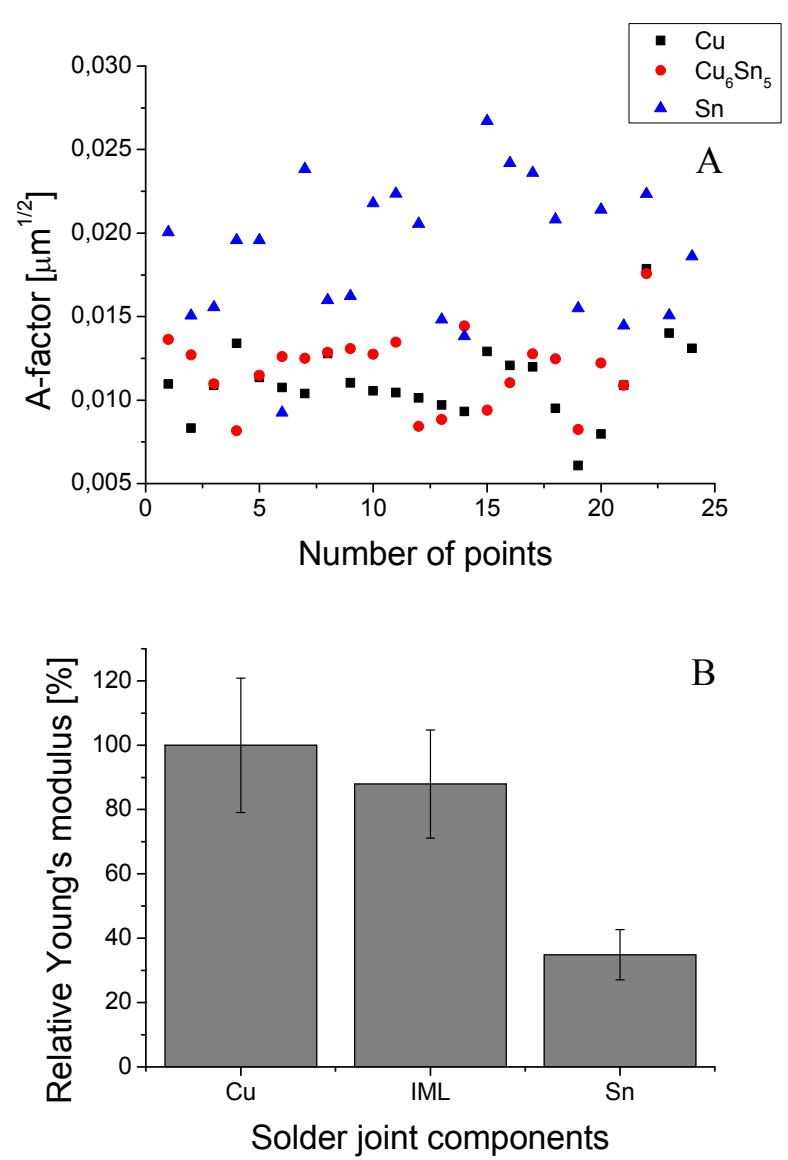

Fig. 3. The fitted A-factors of the contact-mode point spectroscopy measurements at the copper substrate, the IML and the tin region (A) and the representation of the relative Young's modulus compared to the copper substrate (B). 
Instead of calculating the absolute modulus, the relative values are presented in Fig. 3 (B) with regards to the great variance of the spring constant.

Figure 3 shows that the elastic behavior of the IML is more similar to the copper substrate than the $\mathrm{Sn}$ phase. Namely the $\mathrm{Cu}_{6} \mathrm{Sn}_{5} / \mathrm{Cu}$ ratio is $87 \% \pm 17$ and the $\mathrm{Sn} / \mathrm{Cu}$ ratio is $34 \% \pm 7$. The literature values of the Young's modulus vary in a wide range according to the sample preparation methods and the measurement techniques, the published $\mathrm{Cu}_{6} \mathrm{Sn}_{5} / \mathrm{Cu}$ and $\mathrm{Sn} / \mathrm{Cu}$ ratios are $50-97 \%$ and $36-44 \%[4,6]$. Several studies reported the same tendency of the elasticity values as in our measurements; the most of these were obtained by the IIT nanoindentation with a diamond tip [4-6]. However there is a precedent that by using AFM contact-mode point-spectroscopy with a diamond tip the IML phase seemed to be harder than the $\mathrm{Cu}$ substrate [12].

The measured deviation can originate from several effects. One of these is the roughness of the surface caused by the polishing. It can influence the incidence angle of the tip causing the alteration in the effective contact surface area. Another one could be the possible damage of the tip during the measurements. Although great care was taken during the measurement to ensure that the same (undamaged) tip geometry is used for all components, this effect still cannot be neglected.

\subsection{Tip-surface energy dissipation}

To apply the Cleveland's model, tapping-mode imaging was done on the cross-sectioned sample. To

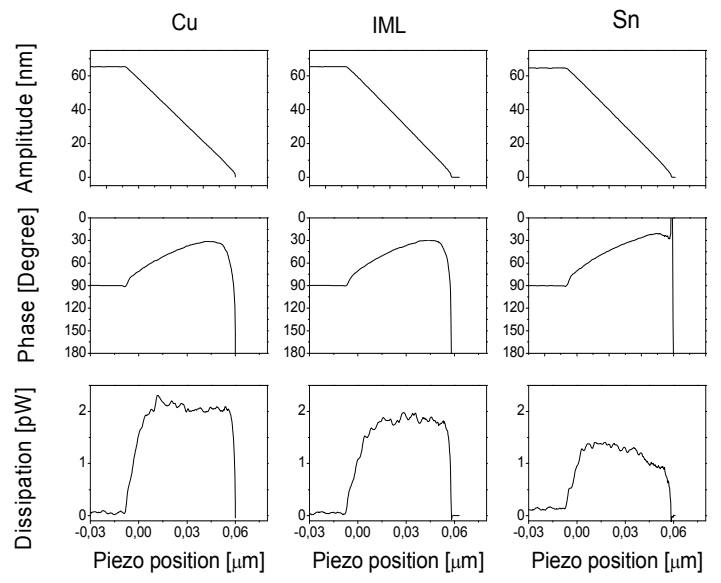

Fig. 4. Measured tapping mode point spectroscopy curves and the calculated dissipation curves at $\mathrm{Cu}$ substrate (left), IML (middle) and Sn-phase (right). set the $\varphi$ phase-lag and $A_{0}$ free-amplitude, tappingmode point-spectroscopy measurements were also done on the different components.

Figure 4 shows typical measured tapping-mode point-spectroscopy curves on the three phases $(\mathrm{Cu}$, $\mathrm{Cu}_{6} \mathrm{Sn}_{5}$ and $\mathrm{Sn}$, respectively) and the calculated dissipation curves based on Eq (4). The measured $Q$ quality factor and the resonant frequencies were 371 and $165 \mathrm{kHz}$ and the nominal spring constant $(5 \mathrm{~N} / \mathrm{m})$ was used for the calculation. It can be seen, that the dissipation curves has a small sensitivity to the variation of the amplitude curves, but the average dissipation of this segment can characterize the sample materials [10]. According to Fig. 4, the biggest dissipation value belongs to the $\mathrm{Cu}$ substrate, the IML layer has a slightly smaller and the lowest value belongs to the Sn-phase, namely $2 \mathrm{pW}, 1.8 \mathrm{pW}$ and $1.25 \mathrm{pW}$. This process characterizes the tip-sample interaction thus using another tip these values can be varied. Nevertheless, during the imaging process the contrast of the calculated dissipation map can characterize the components as it can be seen in the Fig. 5 (C).
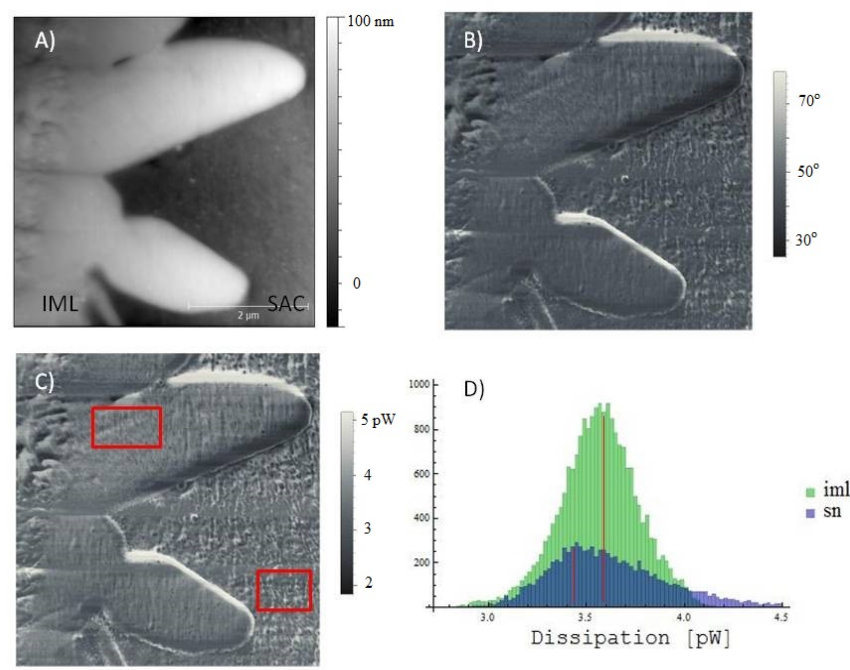

Fig. 5. Tapping-mode AFM topography (A), phase (B) and calculated tip-sample energy dissipation (C) maps with the dissipation histogram (D) of the indicated areas at the IML and at the Sn-phase.

Figure 5 shows the topography and the phase scans with the calculated dissipation map based on the Eq. (3). The measured Q quality factor and the resonant frequency were 315 and $165 \mathrm{kHz}$ and the nominal spring constant $(5 \mathrm{~N} / \mathrm{m})$ was used to the 
calculation. Figure 5 (D) shows the histograms of the areas indicated by red squares in the picture (A), which were marked out in the intermetallic layer and the solder. Although the feedback system was optimized during the scanning (which tried to keep the tapping amplitude as constant) the remaining variations still cause some variation in the measured dissipation, this results in the observable expansion of the spectra. The maximums of the presented histograms are $3.45 \mathrm{pW}$ and $3.6 \mathrm{pW}$, which belong to the intermetallic layer and the tin phase, respectively.

By comparing the tapping-mode pointspectroscopy and tapping imaging results we can see that both the tip parameters and the scanning conditions have a significant influence on the obtained numerical results in the dissipation. We have to state that currently the point-spectroscopy results seem to be more valid and the effects of the scanning and feedback mechanisms are needed be investigated in more detail in the future.

\section{Challenges}

At this point of our research we can identify several challenges which are needed to be solved in order to obtain reliable quantitative information by the application of these proposed methods.

Concerning the determination of Young's modulus the main problem is the reproducibility of the measurements which has two major causes. First, the properties of the tip used for the measurements (spring constant, cone angle etc.) can vary significantly. Several techniques were published for the determination of the spring constant in particular, but most of them can damage the tip or are based on the precise knowledge of the probe's geometry $[13,14]$. We also have to note that the geometry of the tip (half cone angle) can vary during the measurement as the tip becomes eroded. The second problem is the precise determination of the elastic region of the force-curve, in particular, the selection of the endpoints for the fitting. As we discussed in the theory part, there are no accepted models or algorithms for the determination of the last point of the elastic region, and it can be proven, that the selection of this point has a significant influence on the measured Afactors.

Concerning the determination of the tip-surface energy dissipation, the precise knowledge of the cantilever-tip parameters are also critical. Besides this, the generalization of the Cleveland model for imaging requires the identification of the effects of the PID feedback during the constant-amplitude mode scanning. We have seen in Fig 5 that the variations in topography have an effect on the calculated dissipation, but besides this, we think that the setup of the feedback also influences the phase response of the tip during scanning.

Our future work will focus on designing solutions for these challenges to increase the reproducibility and reliability of our results. The investigation of mechanical properties of the much smaller $\mathrm{Ag}_{3} \mathrm{Sn}$ particles will also be in our focus along with other micro-alloyed compounds.

\section{Conclusion}

Different AFM techniques were used to investigate the mechanical properties of a Cu-SAC solder joint interface: contact-mode point-spectroscopy to study the elasticity of the components and tapping-mode imaging to calculate tip-sample dissipation map.

The advantages of these techniques are that the small radius of the tip $(<10 \mathrm{~nm})$ permits a high resolution and fits to the microstructures of the solder; furthermore low forces ( a few $100 \mathrm{nN})$ can be applied, which is another advantage considering the low elasticity of these materials. Although there are several difficulties which were identified during the application of this method, our preliminary results show markable trend, namely that the mechanical properties of the $\mathrm{Cu}_{6} \mathrm{Sn}_{5}$ are closer to the $\mathrm{Cu}$ substrate with a slightly less measured elastic modulus and dissipation. Compared with this, the tin-phase possesses the lowest values in terms of Young's modulus and tip-surface energy dissipation.

\section{ACKNOWLEDGEMENT}

Judit Kámán thanks the support of the ENIAC Joint Undertaking Project. Attila Bonyár is grateful for the support of Bolyai János Scholarship.

\section{REFERENCES}

[1] S.-K. Seo, M. G. Cho, H. M. Lee, W. K. Choi, "Comparison of Sn2.8Ag20In and Sn10Bi10In Solders for Intermediate-Step Soldering", Journal of Electronic Materials, Vol. 35, No. 11, Oct. 2006, pp 1975-1981. 
[2] M. G. Cho, S. K. Kang, D-Y. Shih, H. M. Lee,'Effects of Minor Additions of $\mathrm{Zn}$ in Interfacial Reactions of $\mathrm{Sn}-\mathrm{Ag}-\mathrm{Cu}$ and $\mathrm{Sn}-\mathrm{Cu}$ Solders with Various $\mathrm{Cu}$ Substrates during Thermal Aging", Journal of Electronic Materials, Vol. 35, No. 11 , Oct. 2007,pp 1501-1509.

[3] Y. C. Sohn, J. Yu, S. K. Kang, D. Y. Shih, T. Y. Lee, "Spalling of Intermetallic Compounds During the Reaction Between Lead-Free Solders and Electroless Ni-P Metallization", Journal of Material Research, Vol. 19, No. 8, Mar 2011, pp 2428-2436.

[4] S. Lotfian, J. M. Molina-Aldareguia, K. E. Yazzie, J. Llorca, N. Chawla, "Mechanical Characterization of Lead-Free Sn-Ag-Cu Solder Joints by HighTemperature Nanoindentation", Journal of Electronic Materials, Vol. 42, No. 6, Mar 2013, pp 1085-1091.

[5] Y. Sun, J. Liang, Z.-H. Xu, G. Wang, X. Li, "Nanoindentation for Measuring Individual Phase Mechanical Properties of Lead Free Solder Alloy", Journal of Materials Science; Materials in Electronics, Vol. 19, No. 6, June 2008, pp 514-521.

[6] X. Deng, M. Koopman, N. Chawla, K. K. Chawla, "Young's Modulus of ( $\mathrm{Cu}, \mathrm{Ag})-\mathrm{Sn}$ Intermetallics Measured by Nanoindentation" Materials Science and Engineering, Vol. 364, No. 1-2, Jan. 2004, pp. 240-243.

[7] J.Domke, M. Radmacher. "Measuring the Elastic Properties of Thin Polymer Films with the AFM", Langmuir, Vol. 14, 1998, pp. 3320-3325.

[8] K. A. Melzak, S. Moreno-Flores, K. Yu, J. Kizhakkedathu, J. L. Toca-Herrera, "Rationalized Approach to the Determination of Contact Point in Force-Distance Curves: Application to Polymer Brushes in Salt Solutions and in Water", Microscopy
Research and Technique, Vol. 73, No 10, Oct 2010, 959-964.

[9] S. L. Crick, F. C. P. Yin, “Assessing Micromechanical Properties of Cells with Atomic Force Microscopy: Importance of the Contact Point", Biomechanics and Modeling in Mechanobiology, Springer-Verlag, Vol. 6, No.3, Apr. 2007, 199-210.

[10] J. P. Cleveland, B. Anczykowski, A. E. Schmid, and V. B. Elings, "Energy Dissipation in Tapping-Mode Atomic Force Microscopy", Applied Physics Letters, Vol. 72. No. 20, May 1998, pp. 2613-2615.

[11] B. Anczykowski, B. Gotsmann, H. Fuchs, J. P. Cleveland, "How to measure energy dissipation in dynamic mode atomic force microscopy", Applied Surface Science, Vol. 140, No. 3-4, Febr. 1999, pp. 376-382.

[12] M. A. Monclus, T. J. Young, D. Di Maio, "AFM Indentation Method Used for Elastic Modulus Characterization of Interfaces and Thin Layers", Journal of Materials Science, Vol. 45, No. 12, June 2010, pp. 3190-3197.

[13] T. J. Senden, W. A. Ducker, "Experimental Determination of Spring constant in Atomic Force Microscopy", Langmuir, Vol. 10, No. 4, Apr. 1994, pp. 1003-1004.

[14] R. S: Gates, M. G. Reitsma, J. A. Kramar, J. R. Pratt, "Atomic Force Microscope Cantilever Flexural Stiffness Calibration: Toward a Standard Traceable Method", Journal of Research of the National Institute of Standards and Technology, Vol. 116, No. 4, JulyAugust 2011. 